\title{
Decoding of Motor Imagery Movements from EEG Signals using SpiNNaker Neuromorphic Hardware
}

\author{
Zied Tayeb, Emeç Erçelik, and Jörg Conradt \\ Neuroscientific System Theory, Technical University of Munich, 80333 München, Germany
}

\begin{abstract}
Non-invasive brain machine interfaces (BMIs) on motor imagery movements have been widely studied and used for many years to take advantage of the intuitive link between imagined motor tasks and natural actions. En route to future technical applications of neuromorphic computing, a major current challenge lies in the identification and implementation of brain inspired algorithms to decode recorded signals. Neuromorphic computing is believed to allow real-time implementation of large scale spiking models for processing and computation in non-invasive BMIs. Taking inspiration from the olfactory system of insects, we advance and implement a novel approach to decode and predict imaginary movements from electroencephalogram (EEG) signals. We use a spiking neural network implemented on SpiNNaker (4 chip, 64 cores) neuromorphic hardware. Our work provides a proof of concept for a successful implementation of a functional spiking neural network for decoding two motor imagery (MI) movements on the SpiNNaker system. The approach can be extended to classify more complex MI movements on larger SpiNNaker systems.
\end{abstract}

\section{INTRODUCTION}

Neuromorphic platforms use a different architecture compared to sequential computing, one that mimics brain processing with neurons and synapses. These hardware systems achieve a speedup and support massively parallel data processing in a brain-inspired fashion. In contrast to expectations according to Moore's law, the integration density of digital processors ceased to grow during the last decade and is unlikely to increase again. Hence, neuromorphic architectures are perceived to be a potential solution for this issue, as they express fundamental differences and advantages when compared to conventional computing infrastructure [1].

To meet the challenge of efficient neuromorphic computing, a number of neuromorphic platforms have been and continue to be developed such as SpiNNaker,

All authors are with the Technical University Munich, Germany, Department of Electrical and Computer Engineering, Research Group Neuroscientific System Theory, Center of Competence Neuroengineering. (+4989 28926909, e-mail: zied.tayeb@tum.de)
BrainScales hardware or IBM's True North [2]. SpiNNaker is a novel massively-parallel computer architecture, inspired by the fundamental structure and function of the human brain. Each chip is a multi-core system, consisting of 18 ARM968-based cores and also several internetworking elements and supporting modules. Thus, it supports an emulation of up to 12,000 neurons in biologically plausible real-time per board (SpiNNaker-3) [3] or even about 200,000 neurons per board (SpiNNaker-5).

This work presents a first attempt to use the potential advantages of neuromorphic computing and spiking neural network algorithms to decode brain signals and as per our knowledge no similar work has been published before. This new technology can enhance EEG based brain-machine interface classification performance which still suffers from limited motor imagery (MI) task detection and low accuracy due to non-stationary and non-linear characteristics of the brain signal.

The aim of this work is therefore to decode EEG patterns generated during two MI movements (motor imagery of left and right hand) using a spiking neural network decoder. Here, as the first proof-of-principle, features of EEG signals were extracted and a classifier inspired by the olfactory system of insects was employed and implemented on SpiNNaker to recognize the two different MI movements.

\section{METHODS}

\section{A. Data Description}

Our work is based on experimental data recorded by the Institute for Knowledge Discovery (Laboratory of BrainComputer Interfaces), Graz University of Technology. EEG signals were collected from 9 subjects that were able to perform long and stable motor imagery over a minimum time of 2 -s and data were sampled at $250 \mathrm{~Hz}$. More experimental details are provided in [4].

\section{B. Preprocessing and Feature Extraction}

MI describes the mental rehearsal of a motor task without its execution, such as imagination of squeezing a training ball. These tasks induce a power increase or decrease of EEG amplitudes in certain frequency bands (mainly alpha and beta band) relative to a reference period and are referred 
as event-related (de)synchronization (ERD/ERS) [5]. Hence, three EEG bipolar channels $\mathrm{C} 3, \mathrm{Cz}$ and $\mathrm{C} 4$ (according to the $10 / 20$-system) have been used to analyze these imaginations and to convert them into commands. Epochs of $2000 \mathrm{~ms}$ with $500 \mathrm{~ms}$ pre-stimulus baseline were extracted from the continuous EEG data.

First, all the signals are filtered using a digital Butterworth FIR band pass filter of order 5 in the range $8-30 \mathrm{~Hz}$, because the mu rhythm $(8-13 \mathrm{~Hz})$ and beta band $(14-30 \mathrm{~Hz})$ fall within this range.

Afterwards, band power (BP) features were estimated from the ongoing EEG signal. For each of the three bipolar channels, BP features in 72 frequency bands were calculated using different overlapping narrow bands between 8 and 30 $\mathrm{Hz}$ yielding a total of $216 \mathrm{BP}$ features [4]. As the dimensionality of the extracted EEG features is usually quite high, it is necessary to reduce the dimensionality of the features before we use them. For this purpose, a standard principal component algorithm PCA was applied for dimensionality reduction leading to only 10 features [6].

Moreover, to compare performance against our method a Support Vector Machine linear classifier [7] for two MI was used to test and validate the feature extraction step. A classification accuracy of $77.49 \%$ was obtained. Note that this classifier has been used solely for performance and accuracy comparison with our proposed spiking neural network classifier.

Finally, the features were fed into SpiNNaker and a spiking neural network was trained to classify two-class MI data (left vs right).

\section{Network Architecture and Model Design}

Classical machine learning algorithms have been widely used for EEG signals decoding [8]. However, in order to exploit the native communication protocol within the brain (i.e. discrete events so-called spikes) in brain machine interfaces we need to use an appropriate processing paradigm. Such a paradigm is provided by artificial spiking neural networks which, by using temporal information associated with the spikes to compute, can be used to classify remarkable events in brain signals they represent.

In this paper, we used a classifier network based on a spiking model of the insect olfactory system [9], which has been previously described and investigated in detail in [910]. The model's three-stage architecture consists of (1) an input layer designed to encode multivariate, real-valued data samples into a population-based, positive, bounded, firingrate representation, (2) a decorrelation layer used for filtering and reducing correlation between inputs (where populations are grouped in ensembles that represent the so-called glomeruli in the insect antennal lobe) and finally (3) an association layer that forms a winner-take-all decision circuit through strong lateral inhibition. Figure 1 presents the spiking network model based on the olfactory system of insects.
Spike trains are generated in neurons in the input layer and propagated to the decorelation layer, where projection neuron populations to the excitatory association neurons (orange in the association layer) are adapted by a supervised learning mechanism during training [10].

\section{Implementation on SpiNNaker}

We implemented a spiking neural network that decodes motor imagery movements from recorded EEG signals. The network runs on SpiNNaker neuromorphic hardware and performs its computations in a "purely spike-based fashion" [10]. It incorporates an insect-brain-inspired, three-layer neural architecture. Each of the 10 features from EEG data was encoded in 20 population-coding neurons. The activation of the different population-coded neurons was determined by a Gaussian function and implemented on SpiNNaker as spike source array.

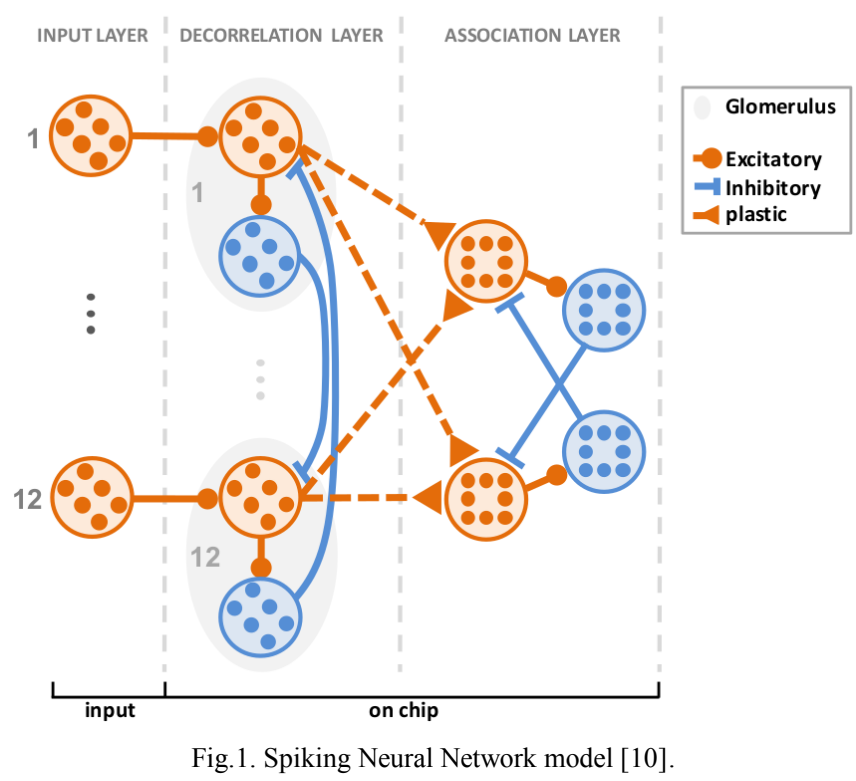

Each spike source array is connected to a population of neurons in the encoding (input) layer. The input layer consists of 200 populations of 10 neurons each, which connect one-to one to the second (decorrelation) layer.

The decorrelation layer is organized in so-called glomeruli, which are functional groups of neurons that exist in the real biological model. Each glomerulus consists of the same number (here $200 * 10$ ) of populations and neurons as the previous one.

Each glomerulus in the decorrelation layer has inhibitory connections with other glomeruli with a probability of $40 \%$. The second layer has excitatory connections to the neurons in the association layer with a probability of $50 \%$.

The association layer consists of two inhibitory and two excitatory glomeruli. Each of the inhibitory population has inhibitory connections to the excitatory one with a $70 \%$ probability. 


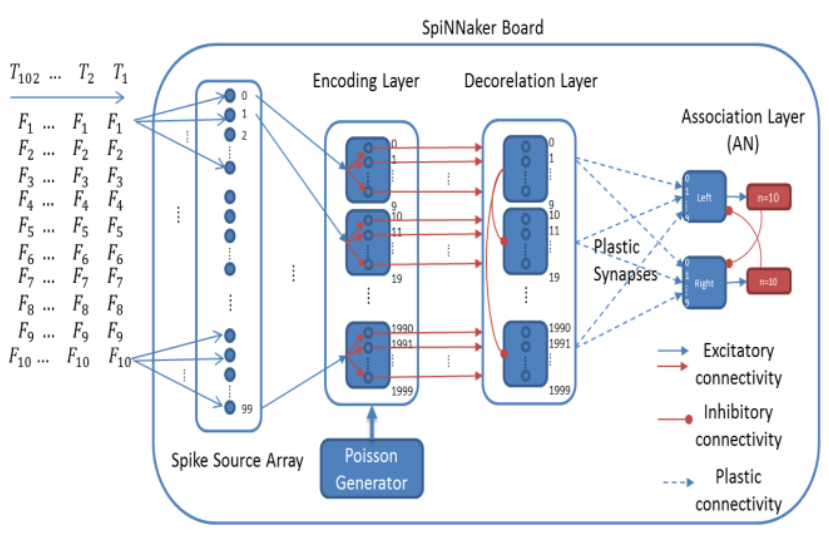

Fig.2. Spiking Neural Network Classifier Implementation on SpiNNaker.

The Spike Source Arrays is connected to the encoding layer by $0.2 \mathrm{nA}$ weights, the connection weights between encoding layer and decorrelation layer are $0.6 \mathrm{nA}$, the inhibitory connection weights inside the decorrelation layer are $0.03 \mathrm{nA}$, the excitatory connections weights from second layer to association layer are $0.08 \mathrm{nA}$, the excitatory connections weights inside the association layer are $0.9 \mathrm{nA}$ and the inhibitory connection weights in the same layer are $0.1 \mathrm{nA}$. The delay of projections was set to $1 \mathrm{~ms}$. Implemented neurons are leaky integrate and fire type neurons, and the synapse models are exponential currentbased synapses. The model parameters used are described in detail in [11]. Furthermore, we used 60 Poisson Generators as noise sources that excite the input layer with $10 \mathrm{~Hz}$ firing rate and $0.02 \mathrm{nA}$ connection weight.

The classification output of the network corresponds to two labeled (left vs. right) populations in the third layer. The difference in the firing rates of each neuron at the decorrelation layer for each class was computed and compared to a fixed threshold. Consequently, we were able to determine the activity of each neuron and to determine the one with higher spike count for a certain class. If this difference exceeds an acceptance threshold for one class, the connection was potentiated with a certain weight value between the associated population in the second layer and the AN population represented the same class in the output layer.

We wish to emphasize that we didn't use directly a Spiketiming dependent plasticity (STDP) to adjust the connections between filtering layer and association layer [11].

\section{RESULTS}

We tested and validated the performance of our spiking neural network classifier implemented on SpiNNaker neuromorphic hardware. The accuracy of our classifier during the validation step was $75 \%$ for a single subject after 120 trials. We wish to emphasize that the massive parallelism of SpiNNaker here is a potential advantage over conventional computing when processing large amounts of data in parallel. Results obtained by the implementation on
SpiNNaker are shown in the upcoming figures and discussed below.

Figure 3 shows the total spiking activity of neurons at the decorelation layer for each class. These neurons present excitatory connections to the two populations AN1 and AN2 of the output layer. This figure shows the number of active neurons in the second layer for the features related to each class separately. As seen, a neuron that is significantly more active for one class makes a connection to the neurons of the same class at the association layer, whereas the less active neurons remain unconnected. Hence, some of the neurons contribute to different classes to make a winner-take all decision and form a winner population.

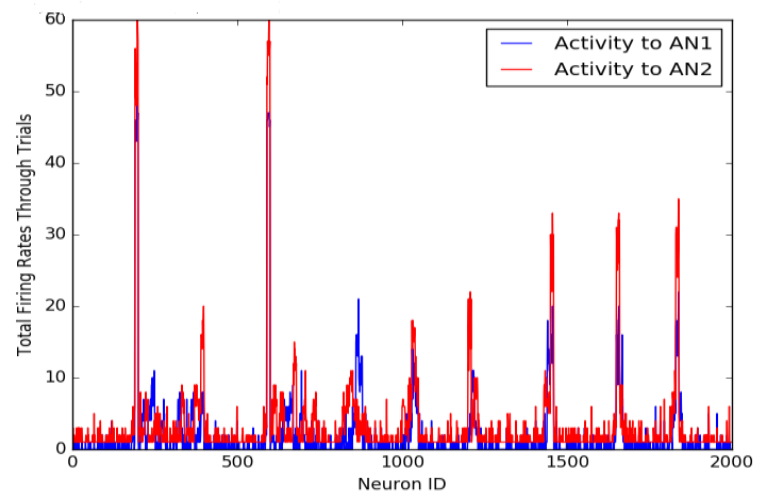

Fig.3. Spiking activity of the neurons in the second layer.

Figure 4 shows the spike trains that we obtained of all neurons in the association layer during a single validation trial. In this figure, vertical lines separate each trial of 200 $\mathrm{ms}$, and dots show the time of occurrence of each spike with its corresponding neuron.

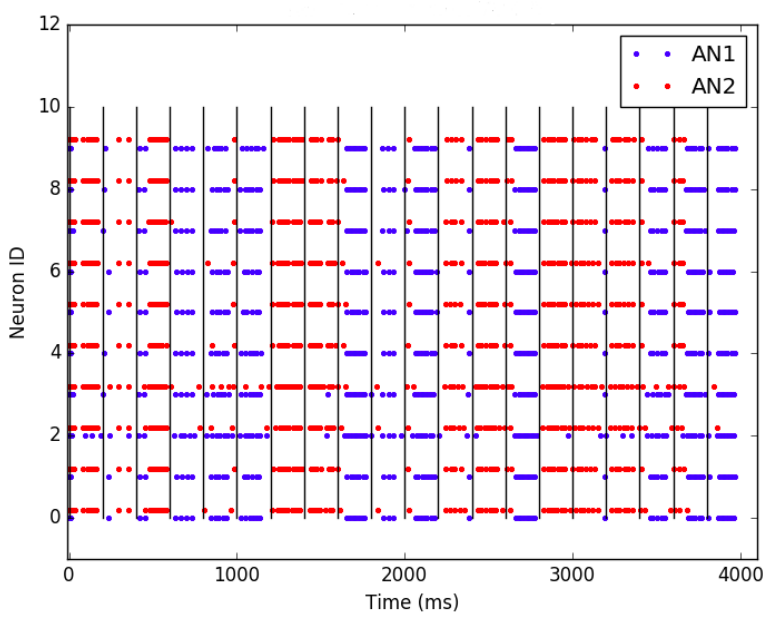

Fig.4. Spiking activity of the neurons in the association layer during validation step (AN1 and AN2 are the two populations in the third layer presenting the two classes).

To test the network's prediction abilities, 20 trials (10 for each class) of non-trained data were used to validate the performance of our classifier. Results are shown in figure 5 . 


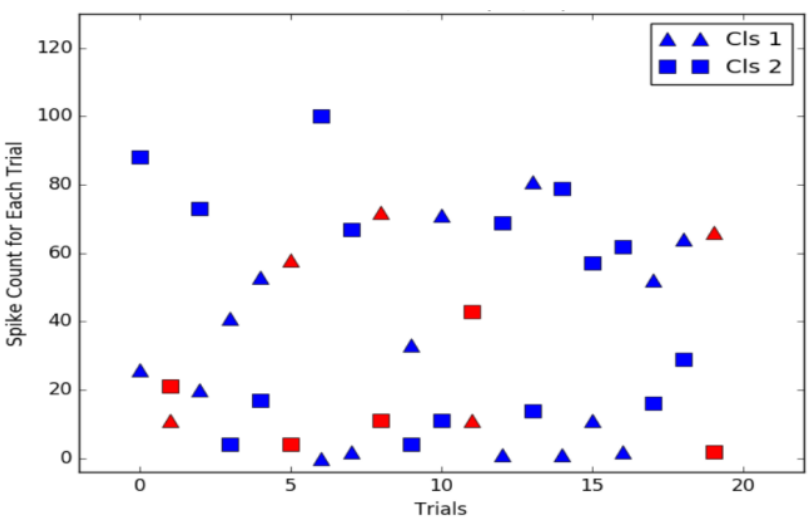

Fig.5. Total spikes at each AN population for each trial during the validation step

The winner for each trial is the AN class that has more spikes. In the figure, the triangles refer to class 1 and the squares refer to class 2. Notably, the blue color presents the correctly classified trials whereas the red color presents the misclassified trials. If the blue square appears above the blue triangle, the class 2 is considered to be the winner for that trial and vice versa. To achieve better classification results, it is utmost important to improve the used method for learning. STDP can be an efficient strategy for our future work.

\section{DISCUSSION}

In our work, an off-line classification of two MI movements from recorded EEG signals has been achieved. Our model uses a spiking neural network implemented on SpiNNaker for time series data classification. The model architecture is inspired by the olfactory system of insects and mimics biological information processing. We achieved $75 \%$ of correctly predicted motor imagery movement on nontrained data.

We wish to point out that the achieved result is still slightly lower than e.g. SVM classifier (77.49\%). As a consequence, we are aiming to improve classification performance by using a biologically plausible STDP method to further train our network. We would like to stress that our current approach presents a proof of concept for decoding non-invasive brain signals using the scalable SpiNNaker spiking neurocomputing hardware, rather than providing a final system. We are currently running the system on the smallest existing SpiNNaker boards, and expect significant improvements in accuracy when scaling-up our spiking model in future research.

As short term goal, we will work on extending the model by incorporating also electromyography (EMG) signals, which will be used for decoding different hand movements.

\section{CONCLUSION}

The above results show that using a spiking neural network classifier can enhance the average imagery classification accuracy in training and evaluation stages with reduced training time and smaller energy and power consumption.
In future work, we are aiming to scale up our model to classify various more complex imaginary movements within the same limb and to move from the classic motor imagery detection (left vs right movement) to decoding complex movement intentions. Furthermore, we are aiming to focus on the kinematic level, where movement direction and hand position or velocity can be decoded from EEG signals and can be used for real-time neuroprosthesis control.

As a long-term research vision, it is likely that the model can be improved also by explicitly including feedback. This will be done by decoding the error-related potential (ErrP), a time-locked potential elicited when actions do not match the users' expectations [9]. Such decoded information from EEG signals will be used as a reward signal to learn and adjust the desired behavior in a closed-loop brain machine interface.

\section{ACKNOWLEDGMENT}

The authors thank Dr. Christoph Richter, Michael Lutter and Stefan Ehrlich for helpful comments and discussions.

\section{REFERENCES}

[1] M. Warldrop, the chips are down for Moore's law, Nature, vol.530, no.7589, pp.144-147, Feb.2016.

[2] Walter, F Röhrbein, F. \& Knoll, Neuromorphic implementation of neurobiological learning algorithms for spiking neural networks. Neural Process, pp. 44-103, 2016.

[3] Furber, Steve B. Gulluppi, Francesco, Temple Steve, Plana, Luis A, The SpiNNaker project, IEEE Transactions on, Vol.102, No.5, 6750072, pp.652-665, 2014.

[4] R. Leeb, F. Lee, C. Keinrath, R. Scherer, H. Bischof and G. Pfurtscheller, "Brain-Computer Communication: Motivation, Aim, and Impact of Exploring a Virtual Apartment," in IEEE Transactions on Neural Systems and Rehabilitation Engineering, vol. 15, no. 4, pp. 473-482, Dec. 2007.

[5] Gernot Müller-Putz and Andreas Schwarz and Joana Pereira and Patrick Ofner", From classic motor imagery to complex movement intention decoding: "The noninvasive Graz-BCI approach",vol 228, 2016, Pages 39-70, progress in Brain Research. Elsevier BV, 2016.

[6] Muhammad Naeem, Clemens Brunner, and Gert Pfurtscheller, "Dimensionality Reduction and Channel Selection of Motor Imagery Electroencephalographic Data," Computational Intelligence and Neuroscience, vol. 2009, pp 1-3, 2009.

[7] A.Sivakami, and S.Shenbaga Devi, analysis of eeg for motor imagery based classification of hand activities, International Journal of Biomedical Engineering and Science (IJBES), Vol. 2, No. 3, July 2015.

[8] Nicolas-Alonso LF, Gomez-Gil J. "Brain Computer Interfaces, a Review". Sensors (Basel, Switzerland), 12(2): pp.1211-1279, 2012.

[9] Michael Schmuker, Thomas Pfeil, and Martin Paul Nawrot, "A neuromorphic network for generic multivariate data classification", PNAS 111 (6), pp. 2081-2086, 2014.

[10] Lungu IA, Riehle A, Nawrot MP, Schmuker M, "Predicting voluntary movements from motor cortical activity with neuromorphic hardware". IBM Journal of Research and Development, in press, 2017.

[11] Diamond Alan, Nowotny Thomas, Schmuker Michael, Comparing Neuromorphic Solutions in Action: Implementing a Bio-Inspired Solution to a Benchmark Classification Task on Three ParallelComputing Platforms, Frontiers in Neuroscience, Vol. 2, 2016.

[12] Francesco Di Gregorioa, Marco Steinhausera, Martin E. Maier, "Error-related brain activity and error awareness in an error classification paradigm", Science Direct, pp. 202-210, 2016. 